\title{
Spike-triggered averages for passive and resonant neurons receiving filtered excitatory and inhibitory synaptic drive
}

\author{
Laurent Badel, ${ }^{1}$ Wulfram Gerstner, ${ }^{1}$ and Magnus J. E. Richardson ${ }^{2, *}$ \\ ${ }^{1}$ Ecole Polytechnique Fédérale de Lausanne, Laboratory of Computational Neuroscience, School of Computer \\ and Communication Sciences and Brain Mind Institute, CH-1015 Lausanne, Switzerland \\ ${ }^{2}$ Warwick Systems Biology Centre, University of Warwick, Coventry CV4 7AL, United Kingdom
}

(Received 22 January 2008; published 22 July 2008)

\begin{abstract}
A path-integral approach is developed for the analysis of spike-triggered average quantities in neurons with voltage-gated subthreshold currents. Using a linearization procedure to reduce the models to the generalized integrate-and-fire form, analytical expressions are obtained in an experimentally relevant limit of fluctuationdriven firing. The influences of voltage-gated channels as well as excitatory and inhibitory synaptic filtering are shown to affect significantly the neuronal dynamics prior to the spike. Analytical forms are given for all relevant physiological quantities, such as the mean voltage triggered to the spike, mean current flowing through voltage-gated channels, and the mean excitatory and inhibitory conductance waveforms prior to a spike. The mathematical results are shown to be in good agreement with numerical simulations of the underlying nonlinear conductance-based models. The method promises to provide a useful analytical tool for the prediction and interpretation of the temporal structure of spike-triggered averages measured experimentally.
\end{abstract}

DOI: 10.1103/PhysRevE.78.011914

PACS number(s): 87.19.1c, 05.10.Gg, 05.40.-a, 87.18.Sn

\section{INTRODUCTION}

Reverse-correlation methods have a long tradition in the neurosciences [1] and are widely used to characterize the response properties of neurons [2,3]. Spike-triggered averages, defined as the mean value of some physiological quantity over the time prior to a spike, provide convenient experimental measures of the salient features of the activity in presynaptic populations that result in a spiking of the postsynaptic neuron. This experimental method is a standard approach used for the mapping of receptive-field structure of retinal ganglion cells [4], neurons of the lateral geniculate nucleus [5], and the primary visual cortex $[6,7]$ as well as different classes of auditory neurons (e.g., $[2,8]$ ).

The spike-triggered average of the intracellular voltage (which will be referred to as STV) is one of the more extensively used quantities: it is accessible in both in vitro and in vivo experiments, and is a function of the combined effects of the temporal structure of the synaptic drive and the response properties of the cell. In an experimental context, a correct inference from the intracellular voltage to the underlying patterns in the synaptic input leading to a spike requires an accurate model of the neuronal response properties: two cells with different voltage-gated-current expression profiles will show significantly different STVs from exposure to the same presynaptic stimulus. Hence, an analytical understanding of the processes that shape the STV and related observables is essential for the understanding of the computational properties of neurons as encoded in their differing thresholded response to fluctuating synaptic drive.

Even in the steady state, extracting the temporal features from nonlinear stochastic systems can be mathematically demanding. For rate-based neuron models, the relation between the spike-triggered average and the properties of the under-

\footnotetext{
*Corresponding author: magnus.richardson@warwick.ac.uk
}

lying model are relatively well understood (e.g., $[9,10]$ ). However, for spiking neuron models this problem is more difficult, and only recently have a number of results been reported for the STV of integrate-and-fire (IF) neurons: a general formula based on mapping the IF model to an escape noise model [11]; an eigenvalue analysis for the leaky IF model of the influence of averaging over isolated and nonisolated spikes [12]; an approximation based on the observation of the time symmetry of the STV for the leaky IF model [13]; analytical formulas in the low-rate limit for the leaky IF neuron and the power-law scaling near threshold $[14,15]$; exact results for the STV of the nonleaky IF model [15]; and a formula [14] for the STV of a two-variable generalized IF neuron. These earlier studies approximated the synaptic fluctuations by a white-noise source. However, excitatory and inhibitory synapses have distinct receptor-inactivation kinetics that lead to a filtering of the input. This feature of synaptic drive could have a potentially significant impact on the temporal patterning of the response, but has been neglected in previous STV analyses.

This paper examines the combined effects on spiketriggered quantities of voltage-gated channels and the filtered synaptic drive typical of excitatory AMPA synapses and inhibitory GABAergic synapses. As will be seen, this involves a significant extension of the path-integral formulation previously used for the white-noise case. Of particular interest will be the effects of subthreshold currents that provide negative feedback, like the hyperpolarization-activated depolarizing current Ih [16] that underlies resonant or oscillatory phenomena [17-22] at the cellular level. Here a generalized integrate-and-fire neuron $[20,21]$ will be used to derive expressions for the time course of the spike-triggered voltage, transmembrane currents, and synaptic conductances. The method identifies the distinct roles played by voltageactivated channels, and excitatory and inhibitory synaptic drive in the run-up to the spike. 


\section{MODEL}

The full nonlinear conductance-based model, which will be integrated numerically, will first be described. Following that, the approximation scheme required to reduce the model to a form which may be tackled mathematically is briefly reviewed and the simplified description, in terms of linear stochastic differential equations, provided.

\section{A. Conductance-based model}

The membrane voltage $V$ of the cell obeys an equation comprising a capacitive term of capacitance $C=1 \mathrm{nF}$ in parallel with a set of subthreshold transmembrane currents $I_{s u b}$ and a synaptic driving current $I_{\text {syn }}$.

$$
C \frac{d V}{d t}+I_{s u b}=I_{s y n}
$$

The spike-generation mechanism is described by a strict threshold at $V_{t h}=-55 \mathrm{mV}$ followed by a reset at a value $V_{r e}$. The form of the reset has a negligible effect on the dynamics because the neuronal response will be treated in the low-rate regime in which memory of the previous spike will have faded by the time of the next one. The effect of the hard threshold and the difference that might be expected were a nonlinear spike-generating current to be included, is considered in the Discussion.

\section{Subthreshold currents}

The subthreshold currents considered here comprise a passive leak current $I_{L}$ of conductance $g_{L}$ and reversal potential $E_{L}=-85 \mathrm{mV}$; a current $I_{P}$ providing positive feedback, similar to the persistent sodium current, with instantaneous activation, a reversal potential $E_{P}=40 \mathrm{mV}$, and a maximal conductance $g_{P}$; and $I_{W}$ an Ih-like hyperpolarizationactivated depolarizing current of maximal conductance $g_{W}$, reversal potential $E_{W}=-20 \mathrm{mV}$ with an activation variable $W$ with voltage-history-dependent dynamics. The instantaneous currents take the form

$$
I_{L}=g_{L}\left(V-E_{L}\right) \text { and } I_{P}=g_{P} P_{\infty}\left(V-E_{P}\right),
$$

with the third current of the form

$$
I_{W}=g_{W} W\left(V-E_{W}\right) \quad \text { with } \tau_{W} \frac{d W}{d t}=W_{\infty}-W,
$$

where $\tau_{W}=75 \mathrm{~ms}$. The activation variables $P_{\infty}$ and $W_{\infty}$ are of the form

$$
A_{\infty}(V)=\left\{1+\exp \left[\left(V-V_{A}\right) / \Delta_{A}\right]\right\}^{-1},
$$

where $V_{A}$ is the voltage at which half the channels are open and $\Delta_{A}$ measures the width of the activation curve. For $I_{W}$ these parameters take the values $V_{W}=-70 \mathrm{mV}, \Delta_{W}$ $=15 \mathrm{mV}$ and for $I_{P}$ these parameters take the values $V_{P}=$ $-60 \mathrm{mV}, \Delta_{P}=15 \mathrm{mV}$. It can be noted that these choices for the activation are relatively broad, so as to allow a meaningful comparison with the linearization procedure. For currents with sharper activation curves the linearization procedure will of course be less accurate, but it will nevertheless pro- vide a good indication of the qualitative response and may be systematically improved upon by going to higher order.

Three parameter sets were chosen to provide neuron models with distinct response profiles: (i) a passive neuron with $g_{L}=0.05$ and $g_{P}=g_{W}=0$, (ii) a neuron with a sag and rebound response with $g_{L}=0.05, g_{W}=0.145$, and $g_{P}=0$, and (iii) a neuron with damped oscillations $g_{L}=0.15, g_{W}=0.15$, and $g_{P}=0.062$. All conductances have units of $\mu \mathrm{S}$. The response of these three neuron models to step current inputs is shown in Fig. 1(A).

\section{Synaptic current}

The synaptic current $I_{s y n}$ is mediated by changes in excitatory $g_{e}(t)$ and inhibitory conductance $g_{i}(t)$. The resulting current [23] can be written

$$
I_{s y n}=g_{e}\left(V-E_{e}\right)+g_{i}\left(V-E_{i}\right),
$$

where $E_{e}=0 \mathrm{mV}$ and $E_{i}=-75 \mathrm{mV}$ are the equilibrium potentials for the two synaptic drives. Using excitation as an example, the total conductance increases by $c_{e}$ each time an excitatory presynaptic spike arrives and inbetween spikes the conductance relaxes back to baseline with a relaxation time scale $\tau_{e}=3 \mathrm{~ms}$ typical for fast excitatory kinetics

$$
\tau_{e} \frac{d g_{e}}{d t}=-g_{e}+c_{e} \tau_{e} \sum \delta\left(t-t_{k}\right)
$$

where the excitatory spike arrival times $t_{k}$ are Poisson distributed with a rate $\mathcal{R}_{e}$ with a similar equation for fast inhibition for which $\tau_{i}=10 \mathrm{~ms}$. It is assumed that excitatory and inhibitory spike trains are uncorrelated.

\section{Monte Carlo simulations}

A forward Euler scheme of time step $d t=10 \mu \mathrm{s}$ or less, was used to integrate the above equation sets [with Poissonian shot noise given in Eq. (6)] for sufficiently long time periods required for the acquisition of 10000 spikes. In Fig. 1(B) we show for the three neuron models defined above a typical voltage trajectory leading to an output spike.

\section{B. Approximation strategy}

In order to reduce the full model into a tractable form, the standard methods [20,21,24-26] of linearization of the activation variables (4) and taking the diffusion limit of the Poisson processes are taken. To perform the linearization, the voltage is expanded around its equilibrium value with only terms that are of first order in the voltage and fluctuating transmembrane conductances retained. As has been shown $[27,28]$ this is equivalent to the Gaussian approximation [29-33] for which the tonic conductance increase and additive fluctuations of the conductance drive are retained, but the multiplicative term is dropped. This is a consistent approximation for the nonthresholded neuron [27,28] because both shot-noise effects and conductance fluctuation effects are neglected. However, shot-noise and conductance fluctuations are retained in the numerical simulations to which the mathematical solutions will be compared. Full details of this procedure can be found in Appendix A. 


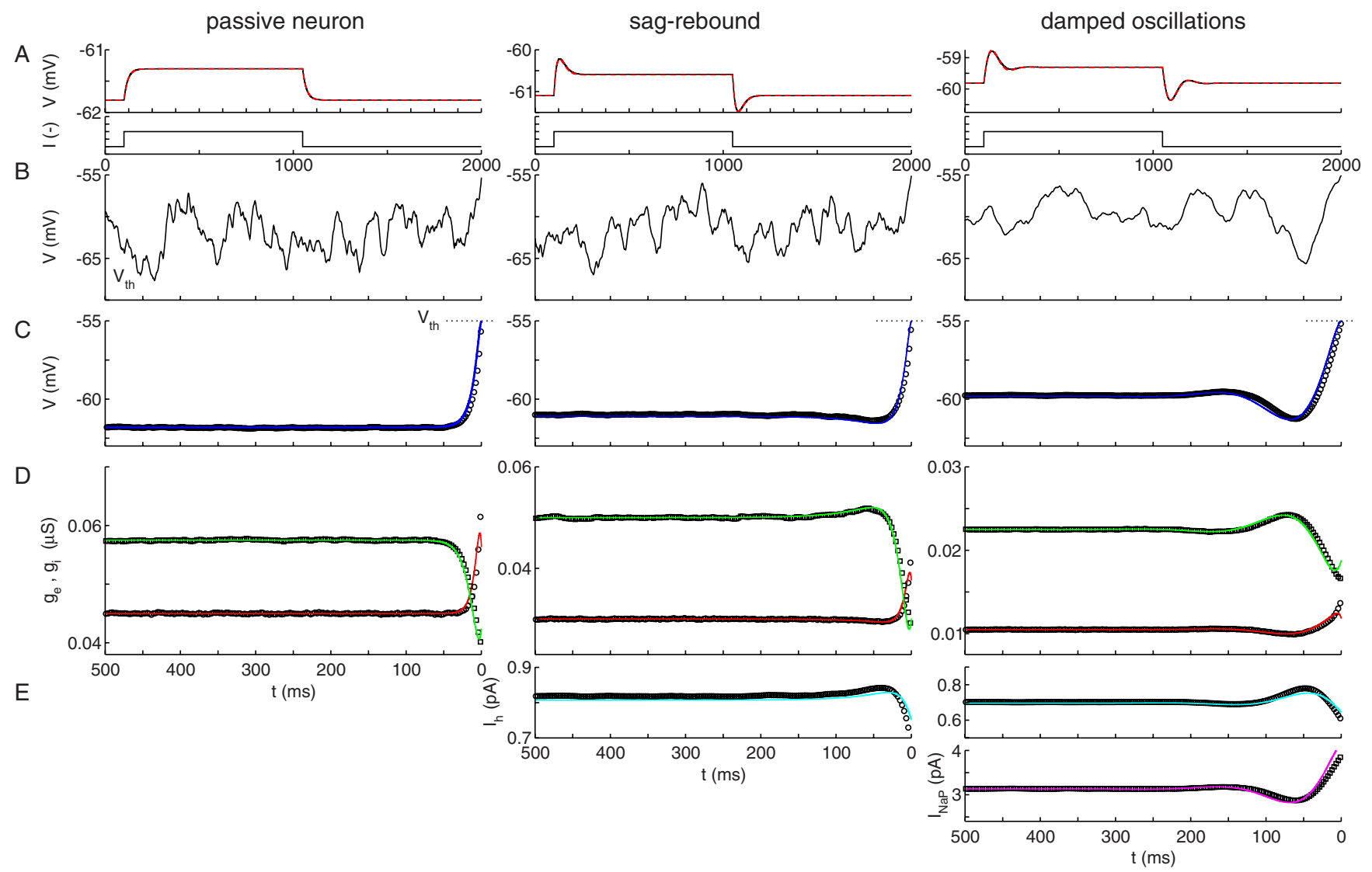

FIG. 1. (Color online) Three different types of neuron model give rise to three qualitatively different spike-triggered averages. (A) Response of the model to a small current pulse causing a steady-state depolarization of about $0.5 \mathrm{mV}$. Three types of response are distinguished: passive decay, sag-rebound, and damped oscillations. (B) Sample voltage trajectories leading to an output spike in the simulations. [(C) and (E)] Comparison of numerical simulations (symbols) of the full conductance-based model to the low-rate theoretical predictions [solid lines, Eqs. (30)-(32), (34)-(36), (B20), and (B21)]. (C) Spike-triggered average voltage. (D) Spike-triggered average synaptic conductances (circles: excitatory conductance; squares: inhibitory conductance). (E) Spike-triggered average values of the intrinsic membrane current (circles: $I_{h}, h$-current; squares: $I_{\mathrm{NaP}}$, persistent sodium current). Parameters for the case of passive decay were rate of arrival of excitatory and inhibitory conductance pulses, $\mathcal{R}_{e}=4.17 \mathrm{kHz}$ and $\mathcal{R}_{i}=1.25 \mathrm{kHz}$; amplitude of conductance pulses, $c_{e}=3.6 \mathrm{nS}$ and $c_{i}=4.6 \mathrm{nS}$; firing rate $r=1.1 \mathrm{~Hz}$. For the sag-rebound case, $\mathcal{R}_{e}=3.06 \mathrm{kHz}, \mathcal{R}_{i}=0.8 \mathrm{kHz}, c_{e}=3.27 \mathrm{nS}, c_{i}=6.26 \mathrm{nS}$, and firing rate $r$ $=1.3 \mathrm{~Hz}$. For damped oscillations, $\mathcal{R}_{e}=4.59 \mathrm{kHz}, \mathcal{R}_{i}=2.81 \mathrm{kHz}, c_{e}=0.76 \mathrm{nS}, c_{i}=0.80 \mathrm{nS}$, and firing rate $r=0.1 \mathrm{~Hz}$.

\section{Simplified model}

The resulting linearized model comprises a set of stochastic differential equations that are the basis of the analytical treatment in this paper.

$$
\begin{gathered}
\tau_{v} \dot{v}=-v-\gamma w+x+y, \\
\tau_{w} \dot{w}=-w+v, \\
\tau_{x} \dot{x}=-x+\sigma_{x} \sqrt{2 \tau_{x}} \xi_{x}(t), \\
\tau_{y} \dot{y}=-y+\sigma_{y} \sqrt{2 \tau_{y}} \xi_{y}(t),
\end{gathered}
$$

where $v$ is the voltage measured from its resting potential, $w$ is proportional to the activation of the $I_{W}$ subthreshold current, and $x$ and $y$ are proportional to the excitatory and inhibitory synaptic fluctuations. The quantities $\xi_{x}$ and $\xi_{y}$ are independent Gaussian white-noise processes with zero mean and delta correlations $\left\langle\xi(t) \xi\left(t^{\prime}\right)\right\rangle=\delta\left(t-t^{\prime}\right)$. The strength of the subthreshold current $I_{W}$ on the voltage is measured by the parameter $\gamma$. A value of $\gamma>0$ results in negative feedback that can lead to a sag response or damped oscillations [20]. All parameters of the linearized model can be related to those of the full nonlinear model, with the details given in Appendix A. The subthreshold dynamics defined by the set of equations (7)-(10) is supplemented by a threshold $v_{t h}$ and reset at $v_{r e}$, which are also measured from the baseline resting potential.

\section{PATH INTEGRAL REPRESENTATION}

In this section we will use a brief review of our previous results [14], for neurons subject to a Gaussian white-noise approximation of synaptic current fluctuations, to introduce the path-integral framework. This also allows for the differences between the cases of unfiltered and filtered synaptic drive to be highlighted more clearly in the next section.

The path-integral formulation for excitatory fluctuations $x(t)$ is used here as an example. It is assumed that the neuronal voltage reaches the threshold at time $t=0$ and so a time 
interval $[-T, 0]$ prior to the spike will be considered. We split this time interval into $N$ bins of size $\Delta_{t}$ such that $t_{k}=-T$ $+k \Delta_{t}$. Integrating Eq. (9) over a time bin and neglecting terms $O\left(\Delta_{t}^{2}\right)$ yields

$$
x_{k+1}=x_{k}-\frac{\Delta_{t}}{\tau_{x}} x_{k}+\sigma_{x} \sqrt{\frac{\Delta_{t}}{\tau_{v}}} \psi_{k},
$$

where $\psi_{k}$ is a Gaussian random number of zero mean and unit variance. The distribution of $x_{k+1}$ at $t_{k+1}$, given $x_{k}$ at $t_{k}$, will also be Gaussian, and so the probability density of finding a discrete-time trajectory with values $\left\{x_{k}\right\}$ can be written

$$
\mathcal{P}\left(\left\{x_{k}\right\}\right) \propto \exp \left(-\sum_{k=-N}^{1} \frac{\psi_{k}^{2}}{2}\right)=\exp \left[-\mathcal{S}\left(\left\{x_{k}\right\}\right)\right] .
$$

The quantity $\mathcal{S}\left(\left\{x_{k}\right\}\right)$ on the right-hand side of Eq. (12) can be rewritten by solving Eq. (11) for $\psi_{k}$, yielding

$$
\mathcal{S}\left(\left\{x_{k}\right\}\right)=\frac{1}{4 \sigma_{x}^{2}} \sum_{k=-N}^{1} \frac{\Delta_{t}}{\tau_{x}}\left(\tau_{x} \frac{x_{k+1}-x_{k}}{\Delta_{t}}+x_{k}\right)^{2} .
$$

For calculational purposes it proves convenient to take the continuum limit $\Delta_{t} \rightarrow 0$ to yield

$$
\mathcal{S}[x(t)]=\frac{1}{4 \sigma_{x}^{2} \tau_{x}} \int_{-T}^{0} d t\left(\tau_{x} \dot{x}+x\right)^{2} d t .
$$

This quantity is called the action [34] of the path integral. For the case of relatively weak fluctuations considered here, $\sigma_{x}$ is small, and so the probability density will be strongly peaked around the trajectory that minimizes the integral on the right-hand side of Eq. (14). The extremizing trajectory may be found using standard methods of the calculus of variations. In general, the limit $T \rightarrow \infty$ will be taken so that trajectories will be considered that came out of a steady-state ensemble in the distant past. This is an acceptable approximation for neurons that fire with a typical period that is considerably longer than the intrinsic time constants of the membrane dynamics.

\section{A. Neuronal response to Gaussian white-noise synaptic fluctuations}

The spike-triggered average trajectory has been previously analyzed, in the weak-noise limit, for neurons with a passive response $[14,15,35]$ and with linear membranes exhibiting a sag or damped oscillatory response [14] when the synaptic fluctuations are modeled using Gaussian white noise. These results are now briefly reviewed, using the formalism demonstrated in the derivation of Eq. (14).

\section{Passive membranes}

The case of a passive membrane with a white-noise drive is given by Eq. (7) with $\gamma=0$ and $x+y$ replaced by $\sigma_{v} \sqrt{2 \tau_{v}} \xi(t)$, where $\sigma_{v}^{2}$ is the variance of the voltage in absence of threshold. This equation is of the OrnsteinUhlenbeck form and so has an action identical in form to that given by Eq. (14) but with the assignment $x \rightarrow v$ and $T \rightarrow \infty$.

$$
\mathcal{S}=\frac{1}{4 \sigma_{v}^{2} \tau_{v}} \int_{-\infty}^{0} d t\left(\mathcal{L}_{v} v\right)^{2}
$$

where the operator and its adjoint are defined as

$$
\mathcal{L}_{z}=1+\tau_{z} \frac{d}{d t} \quad \text { and } \quad \mathcal{L}_{z}^{\dagger}=1-\tau_{z} \frac{d}{d t}
$$

for any quantity $z$. From the calculus of variations the minimizing trajectory obeys

$$
\mathcal{L}_{v} \mathcal{L}_{v}^{\dagger} v=0 \text { yielding } v(t)=v_{t h} e^{t / \tau_{v}} .
$$

This result $[14,15,35]$ provides a good approximation to the spike-triggered average voltage in the low-rate, fluctuationdriven firing regime. However, near threshold $v_{t h}$ a boundary effect intervenes that is not captured by the low-rate approximation. Near the boundary the trajectory can be shown [14] to take the form of a power law for $t \rightarrow 0, t<0$,

$$
v(t) \simeq v_{t h}-\sigma_{v} \sqrt{\frac{16|t|}{\pi \tau_{v}}} .
$$

This singular behavior is due to the interaction of the white noise with an absorbing boundary and is also observed in the absence of leak currents [15].

\section{Linearized, quasiactive membranes}

Biologically important membrane response properties such as a sag-rebound, associated with the Ih current, or damped oscillations have been modeled [14,20,21] using Eqs. (7) and (8) with $\gamma>0$ and the Gaussian white-noise approximation with the replacement $x+y \rightarrow \sigma \sqrt{2 \tau_{v}} \xi(t)$. The action in this case takes the form

$$
\mathcal{S}=\frac{1}{4 \sigma^{2} \tau_{v}} \int_{-\infty}^{0} d t\left(\mathcal{L}_{v} v+\gamma w\right)^{2} .
$$

The minimization problem becomes straightforward when Eq. (8) is rearranged to yield $v=\mathcal{L}_{w} w$ and substituted into the integral to give the integrand in the form $\left(\mathcal{L}_{v} \mathcal{L}_{w} w+\gamma w\right)^{2}$. The minimizing trajectory satisfies

$$
\left(\mathcal{L}_{v} \mathcal{L}_{w}+\gamma\right)\left(\mathcal{L}_{v}^{\dagger} \mathcal{L}_{w}^{\dagger}+\gamma\right) w=0 .
$$

This fourth-order linear equation has exponential solutions with eigenvalues $\pm \lambda_{1}$ and $\pm \lambda_{2}$, where

$$
\lambda_{1,2}=-\frac{1}{2} \frac{\left(\tau_{v}+\tau_{w}\right) \pm \sqrt{\left(\tau_{v}-\tau_{w}\right)^{2}-4 \tau_{v} \tau_{w} \gamma}}{\tau_{v} \tau_{w}} .
$$

On imposing the boundary conditions $v(t=0)=v_{t h}$, further optimization over the possible values of $w$ at threshold yields the spike-triggered average voltage as

$$
\begin{aligned}
v(t)= & \frac{v_{\theta}}{\lambda_{1} \lambda_{2} \tau_{w}^{2}+1} \\
& \quad \times\left(\frac{\lambda_{2}\left(\lambda_{1}^{2} \tau_{w}^{2}-1\right)}{\lambda_{1}-\lambda_{2}} e^{-\lambda_{1} t}+\frac{\lambda_{1}\left(\lambda_{2}^{2} \tau_{w}^{2}-1\right)}{\lambda_{2}-\lambda_{1}} e^{-\lambda_{2} t}\right) .
\end{aligned}
$$

Though the action in Eq. (19) comprised two system variables, as shown in Eq. (20) it was possible to substitute for 
one of the variables $(v)$ so as to rewrite the action in terms of a single state variable. This simple approach is not possible for the case of two independent noise sources with distinct filter constants, and therefore a more involved method needs to be developed, as will now be shown.

\section{FILTERED SYNAPTIC DRIVE}

In order not to overly obscure the reading of the basic framework required to treat the case of two noise sources with distinct filtering constants, the bulk of the calculation details are to be found in Appendix B. We will now proceed with the case of the passive membrane, and then extend these results to the case of linearized, quasiactive membranes.

\section{A. Passive membranes with filtered noise sources}

The passive model $(\gamma=0)$ with filtered synaptic drive, Eqs. (7)-(10), has an action of the form

$$
\mathcal{S}(x, y)=\frac{1}{4} \int_{-\infty}^{0}\left\{\frac{1}{\sigma_{x}^{2} \tau_{x}}\left(\mathcal{L}_{x} x\right)^{2}+\frac{1}{\sigma_{y}^{2} \tau_{y}}\left(\mathcal{L}_{y} y\right)^{2}\right\} d t,
$$

where the two synaptic fluctuations $x, y$ are assumed to be independent. It is now no longer possible to reduce the problem to a variational minimization over a single variable; the action functional must be minimized over the set of paired trajectories $\{x(t), y(t)\}$, which trigger an output spike exactly at $t=0$, i.e., which led to $v(0)=v_{\theta}$. Using Eq. (7), this condition can be rewritten as an integral constraint

$$
\mathcal{G}(x, y)=\int_{-\infty}^{0} e^{t / \tau_{v}}[x(t)+y(t)] d t-\tau_{v} v_{\theta}=0 .
$$

Following the standard methods of the calculus of variations (see Appendix B) the solution to this minimization problem satisfies the system of differential equations

$$
\begin{aligned}
& \mathcal{L}_{x} \mathcal{L}_{x}^{\dagger} x=\Lambda e^{t / \tau_{v}}, \\
& \mathcal{L}_{y} \mathcal{L}_{y}^{\dagger} y=\Lambda e^{t / \tau_{v}},
\end{aligned}
$$

where $\Lambda$ is the Lagrange multiplier associated with the threshold condition. This gives

$$
\begin{aligned}
& x(t)=c_{1} e^{t / \tau_{v}}+c_{2} e^{t / \tau_{x}}, \\
& y(t)=d_{1} e^{t / \tau_{v}}+d_{2} e^{t / \tau_{y}} .
\end{aligned}
$$

The four constants $c_{1}, c_{2}, d_{1}$, and $d_{2}$ can be determined by inserting these expressions back into Eqs. (24) and (25) and solving the resulting algebraic minimization problem (see Appendix B). The resulting form of the mean excitatory synaptic fluctuations in the run-up to the spike is

$$
x(t)=\theta_{x}\left(\frac{2 \tau_{v}}{\tau_{v}-\tau_{x}} e^{t / \tau_{v}}+\frac{\tau_{x}+\tau_{v}}{\tau_{x}-\tau_{v}} e^{t / \tau_{x}}\right),
$$

where

$$
\theta_{x}=v_{\theta}\left(1+\frac{\sigma_{y}^{2} \tau_{y}\left(\tau_{v}+\tau_{x}\right)}{\sigma_{x}^{2} \tau_{x}\left(\tau_{v}+\tau_{y}\right)}\right)^{-1} .
$$

A similar expression for the inhibitory fluctuations is obtained by switching the indices $x$ and $y$. Then, from Eqs. (7),
(30), and (31), the time course of the spike-triggered average membrane voltage is found to be

$$
\begin{aligned}
v(t)= & \theta_{x}\left(\frac{\tau_{v}}{\tau_{v}-\tau_{x}} e^{t / \tau_{v}}+\frac{\tau_{x}}{\tau_{x}-\tau_{v}} e^{t / \tau_{x}}\right) \\
& +\theta_{y}\left(\frac{\tau_{v}}{\tau_{v}-\tau_{y}} e^{t / \tau_{v}}+\frac{\tau_{y}}{\tau_{y}-\tau_{v}} e^{t / \tau_{y}}\right) .
\end{aligned}
$$

The theoretical results of Eqs. (30)-(32) are in good agreement with numerical simulations of the passive neuron model [Fig. 2(C)]. Equation (32) allows for an interpretation of the quantities $\theta_{x}$ and $\theta_{y}$. At the point of the spike, when $t=0$ the voltage $v_{t h}$ is equal to their sum. Hence, $\theta_{x}$ and $\theta_{y}$ measure the contribution of excitatory and inhibitory fluctuations to the reaching of the threshold. Both these terms are positive, but the correct interpretation is that the positive inhibitory contribution comes from fluctuations in inhibition that momentarily weaken the baseline inhibition. It can further be noted that because $\theta_{x} / \theta_{y} \propto \sigma_{x}^{2} / \sigma_{y}^{2}$, the relative contribution of the synaptic fluctuations scales with the ratio of their variances.

The form of the voltage equation (32) shows explicitly the effect of the filtering of the noise. If the limit of $\tau_{x}, \tau_{y} \rightarrow 0$ is taken, the white-noise result Eq. (17) is recovered. However, given that the values for the membrane time constant for cortical cells are in the range 10-40 $\mathrm{ms}$ and that inhibitory filtering has a time constant of $10 \mathrm{~ms}$, the filtering can be significant. This effect is further compounded when conditions of high synaptic conductance are considered for which the effective membrane time constant $\tau_{v}$ can be reduced to as little as $5 \mathrm{~ms}$ [36]. Under such circumstances the synaptic contribution (prefactor of the exponential with the $\tau_{y}$ decay) is twice as large as the contribution from the membrane dynamics (prefactor of the exponential with the $\tau_{v}$ decay).

\section{B. Linearized active membranes with filtered noise}

The case of quasiactive membranes is now studied, in which an additional subthreshold current is present $(\gamma>0)$. The calculation proceeds as for the passive case, but with the threshold condition now taking the form

$$
\int_{-\infty}^{0}\left(p_{1} e^{-\lambda_{1} t}+p_{2} e^{-\lambda_{2} t}\right)[x(t)+y(t)] d t-\tau_{v} v_{\theta}=0 .
$$

The eigenvalues $\lambda_{1}$ and $\lambda_{2}$ are given by Eq. (21), and the coefficients $p_{1}$ and $p_{2}$ are related to the change of coordinates that diagonalizes the linear system (7) and (8) (see Appendix $\mathrm{B}$ for details). The stationarity condition (Euler-Lagrange equation) gives the following form for the synaptic conductances:

$$
\begin{aligned}
& x(t)=c_{1} e^{-\lambda_{1} t}+c_{2} e^{-\lambda_{2} t}+c_{3} e^{t / \tau_{x}}, \\
& y(t)=d_{1} e^{-\lambda_{1} t}+d_{2} e^{-\lambda_{2} t}+d_{3} e^{t / \tau_{y}} .
\end{aligned}
$$

The constants $c_{k}$ and $d_{k}$ can be found in closed form (see Appendix B for details). We obtain for the synaptic conductances, 


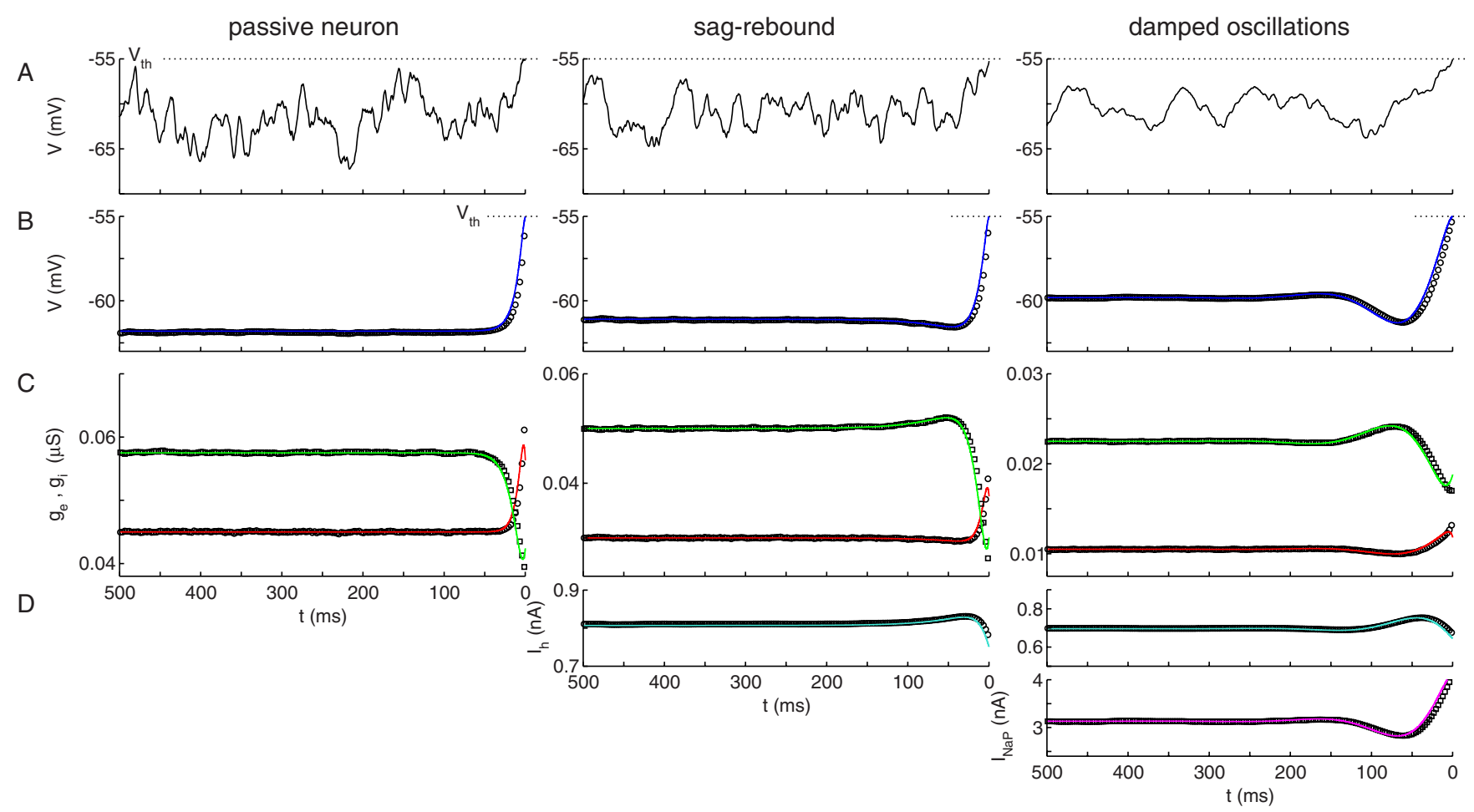

FIG. 2. (Color online) Spike-triggered averages in the simulations and in the theory. Numerical simulations of the simplified model (symbols) are compared to the low-rate theoretical predictions (solid lines). The model is defined by Eqs. (7)-(10), and the theoretical predictions were calculated using Eqs. (30)-(32) for the passive case, and Eqs. (34)-(36), (B20), and (B21) for the two other cases. (A) Sample voltage trajectories leading to an output spike in the simulations. (B) Spike-triggered average voltage. (C) Spike-triggered average synaptic conductances (circles: excitatory conductance; squares: inhibitory conductance). (D) Spike-triggered average values of the intrinsic membrane current (circles: $I_{h}, h$-current; squares: $I_{\mathrm{NaP}}$, persistent sodium current). The parameters of the reduced model were derived from the full model in Fig. 1. For all three cases, $\tau_{w}=75 \mathrm{~ms}$. For the case of passive decay, $\tau_{v}=6.56 \mathrm{~ms}, \gamma=0, \sigma_{x}=3.65 \mathrm{mV}, \sigma_{y}=2.13 \mathrm{mV}$, and firing rate $r=1.0 \mathrm{~Hz}$. For the sag-rebound case, $\tau_{v}=6.68 \mathrm{~ms}, \gamma=0.62, \sigma_{x}=2.86 \mathrm{mV}, \sigma_{y}=2.41 \mathrm{mV}$, and firing rate $r=1.0 \mathrm{~Hz}$. For damped oscillations, $\tau_{v}=39.02 \mathrm{~ms}, \gamma=3.20, \sigma_{x}=4.67 \mathrm{mV}, \sigma_{y}=3.53 \mathrm{mV}$, and firing rate $r=0.2 \mathrm{~Hz}$.

$$
\begin{aligned}
x(t)= & \theta_{x}\left(\frac{\left(\tau_{v}+\tau_{w}\right) \lambda_{1} \lambda_{2}\left(1-\tau_{x} \lambda_{1}\right)\left(1-\tau_{x} \lambda_{2}\right)}{1+\tau_{w}^{2} \lambda_{1} \lambda_{2}-\tau_{x}\left(\lambda_{1}+\lambda_{2}\right)}\right) \\
& \times\left(\frac{2\left(1+\tau_{w} \lambda_{1}\right)}{\left(1-\tau_{x}^{2} \lambda_{1}^{2}\right)\left(\lambda_{1}-\lambda_{2}\right)} e^{-\lambda_{1} t}+\frac{2\left(1+\tau_{w} \lambda_{2}\right)}{\left(1-\tau_{x}^{2} \lambda_{2}^{2}\right)\left(\lambda_{2}-\lambda_{1}\right)} e^{-\lambda_{2} t}\right. \\
& \left.+\frac{\left(\tau_{x}-\tau_{w}\right)}{\left(1+\tau_{x} \lambda_{1}\right)\left(1+\tau_{x} \lambda_{2}\right)} e^{t / \tau_{x}}\right)
\end{aligned}
$$

with the eigenvalues $\lambda_{1}, \lambda_{2}$ given by Eq. (21), and

$$
\begin{aligned}
\theta_{x}= & v_{\theta}\left(1+\frac{\sigma_{y}^{2} \tau_{y}\left(1-\tau_{x} \lambda_{1}\right)\left(1-\tau_{x} \lambda_{2}\right)}{\sigma_{x}^{2} \tau_{x}\left(1-\tau_{y} \lambda_{1}\right)\left(1-\tau_{y} \lambda_{2}\right)}\right. \\
& \left.\times \frac{1+\tau_{w}^{2} \lambda_{1} \lambda_{2}-\tau_{y}\left(\lambda_{1}+\lambda_{2}\right)}{1+\tau_{w}^{2} \lambda_{1} \lambda_{2}-\tau_{x}\left(\lambda_{1}+\lambda_{2}\right)}\right)^{-1}
\end{aligned}
$$

The voltage trajectory can be expressed as $v(t)=v_{x}(t)+v_{y}(t)$, where

$$
\begin{aligned}
v_{x}(t)= & \theta_{x}\left(\frac{\left(1-\tau_{x} \lambda_{1}\right)\left(1-\tau_{x} \lambda_{2}\right)}{1+\tau_{w}^{2} \lambda_{1} \lambda_{2}-\tau_{x}\left(\lambda_{1}+\lambda_{2}\right)}\right) \\
& \times\left(\frac{\lambda_{2}\left(\lambda_{1}^{2} \tau_{w}^{2}-1\right)}{\left(1-\tau_{x}^{2} \lambda_{1}^{2}\right)\left(\lambda_{1}-\lambda_{2}\right)} e^{-\lambda_{1} t}\right. \\
& +\frac{\lambda_{1}\left(\lambda_{2}^{2} \tau_{w}^{2}-1\right)}{\left(1-\tau_{x}^{2} \lambda_{2}^{2}\right)\left(\lambda_{2}-\lambda_{1}\right)} e^{-\lambda_{2} t} \\
& \left.+\frac{\lambda_{1} \lambda_{2}\left(\lambda_{1}+\lambda_{2}\right)\left(\tau_{w}^{2}-\tau_{x}^{2}\right) \tau_{x}}{\left(1-\tau_{x}^{2} \lambda_{1}^{2}\right)\left(1-\tau_{x}^{2} \lambda_{2}^{2}\right)} e^{t / \tau_{x}}\right),
\end{aligned}
$$

and $v_{y}(t)$ is obtained by switching the indices $x$ and $y$. By taking the limit $\tau_{x}, \tau_{y} \rightarrow 0$, it can be verified that this expression is consistent with the result for unfiltered synaptic fluctuations, Eq. (22). For $\gamma \rightarrow 0$, we have $\lambda_{1} \rightarrow-1 / \tau_{v}$ and $\lambda_{2}$ $\rightarrow-1 / \tau_{w}$, and the result for the passive membrane, Eq. (32), is recovered.

Similarly to the passive case, it can be seen that the two quantities $\theta_{x}$ and $\theta_{y}$ measure the contributions of excitatory and inhibitory conductance fluctuations to the total membrane depolarization. Since the eigenvalues $\lambda_{1}$ and $\lambda_{2}$ have a negative real part, it follows that these two contributions are 

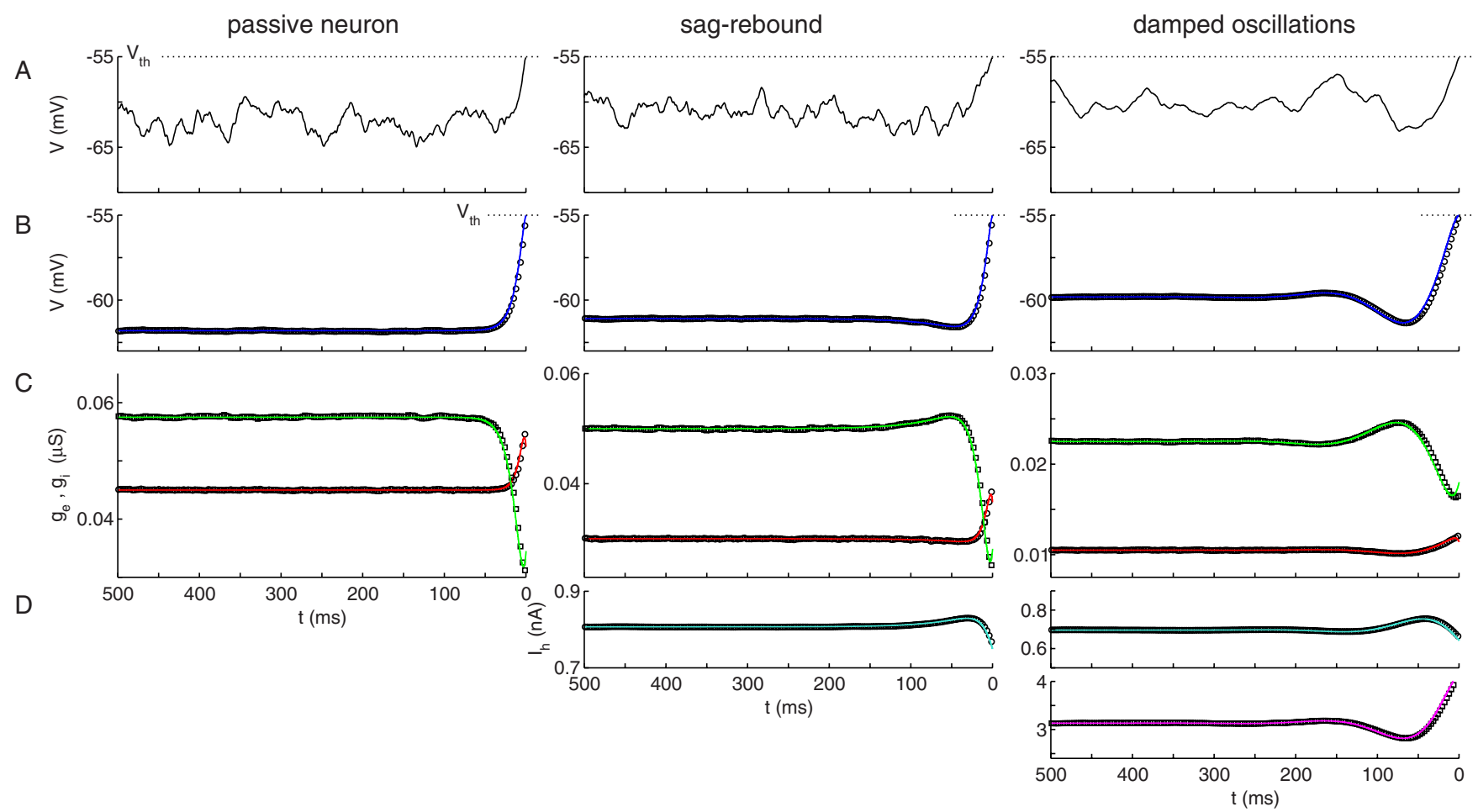

FIG. 3. (Color online) Spike-triggered averages at very low firing rates. The theoretical predictions [solid lines, Eqs. (30)-(32), (34)-(36), (B20), and (B21)] are compared with numerical simulations of the simplified model (symbols) for a firing rate of $10^{-4} \mathrm{~Hz}$. (A) Sample voltage trajectories leading to an output spike in the simulations. (B) Spike-triggered average voltage. (C) Spike-triggered average synaptic conductances (circles: excitatory conductance; squares: inhibitory conductance). (D) Spike-triggered average values of the intrinsic membrane current (circles: $I_{h}, h$-current; squares: $I_{\mathrm{NaP}}$, persistent sodium current). The parameters are the same as in Fig. 2 , except $\sigma_{x}$ $=1.54 \mathrm{mV}, \sigma_{y}=1.37 \mathrm{mV}$ (decay), $\sigma_{x}=1.39 \mathrm{mV}, \sigma_{y}=1.31 \mathrm{mV}$ (sag-rebound), $\sigma_{x}=2.33 \mathrm{mV}$, and $\sigma_{y}=2.36 \mathrm{mV}$ (damped oscillations).

positive: the voltage run-up to the spike is due to both an increase in excitation and a decrease in inhibition, so that both excitatory and inhibitory fluctuations take part in firing the cell. Furthermore, the relative contribution of each conductance again scales with the ratio of the noise strengths $\theta_{x} / \theta_{y} \propto \sigma_{x} / \sigma_{y}$.

\section{Comparison of the theory with numerical simulations of the full model}

Figure 1 compares the theoretical results of the preceding section with numerical simulations of the full conductancebased model defined by Eqs. (1)-(6). The theoretical spiketriggered average voltage and conductances were obtained using Eqs. (30)-(32) for the decay case (left column in Fig. 1), and Eqs. (34)-(36) for the two other cases (sag-rebound and damped oscillations). For the latter we also show in Fig. 1(D) the spike-triggered average of the voltage-activated subthreshold currents and compare it to the theoretical results of Eqs. (B20) and (B21) given in Appendix B.

The data shows a good agreement between theory and simulation, particularly given the fact that the full model incorporates two nonlinear voltage-gated subthreshold currents and retains both the conductance and shot-noise aspects of the synaptic drive, whereas the theoretical calculations were carried out with the linearized model and within the Gaussian approximation to synaptic fluctuations. The bulk part of the spike-triggered average is well approximated for all three types of subthreshold dynamics. The theory also captures the time course of the excitatory and inhibitory synaptic conductances in the run-up to the spike: in the lowfiring rate regime the average trajectory preceding a spike is close to the trajectory of highest probability calculated from the variational approach.

The major discrepancy occurs in a small temporal region, just before the spike threshold is crossed. In the last few milliseconds $(<5 \mathrm{~ms})$ before the spike is triggered, the synaptic conductances in the theory begin to relax back to their baseline value - a feature that is not seen in the simulations. The reason for this is that in the extremization procedure the voltage derivative $d v / d t$ must be zero at threshold because the most likely trajectory during weak noise is the one which glances threshold [this can be seen directly from the analytical expressions of the STV, Eqs. (32) and (36)]. This progressive slowing of the membrane potential also causes a slight shift of the STV along the time axis, which is most visible in the case of damped oscillations (right column in Fig. 1). This effect does not stem from the linearization of the voltagegated currents or the approximation of the synaptic input, as it is also seen in numerical simulations of the reduced model (see Fig. 2). Rather, it is due to the fact that the theory is asymptotically exact in the limit of vanishing firing rate, and that the conditions in the numerical simulations depart slightly from this limit. Indeed, a clear convergence is seen as the firing rate approaches zero (Fig. 3). 


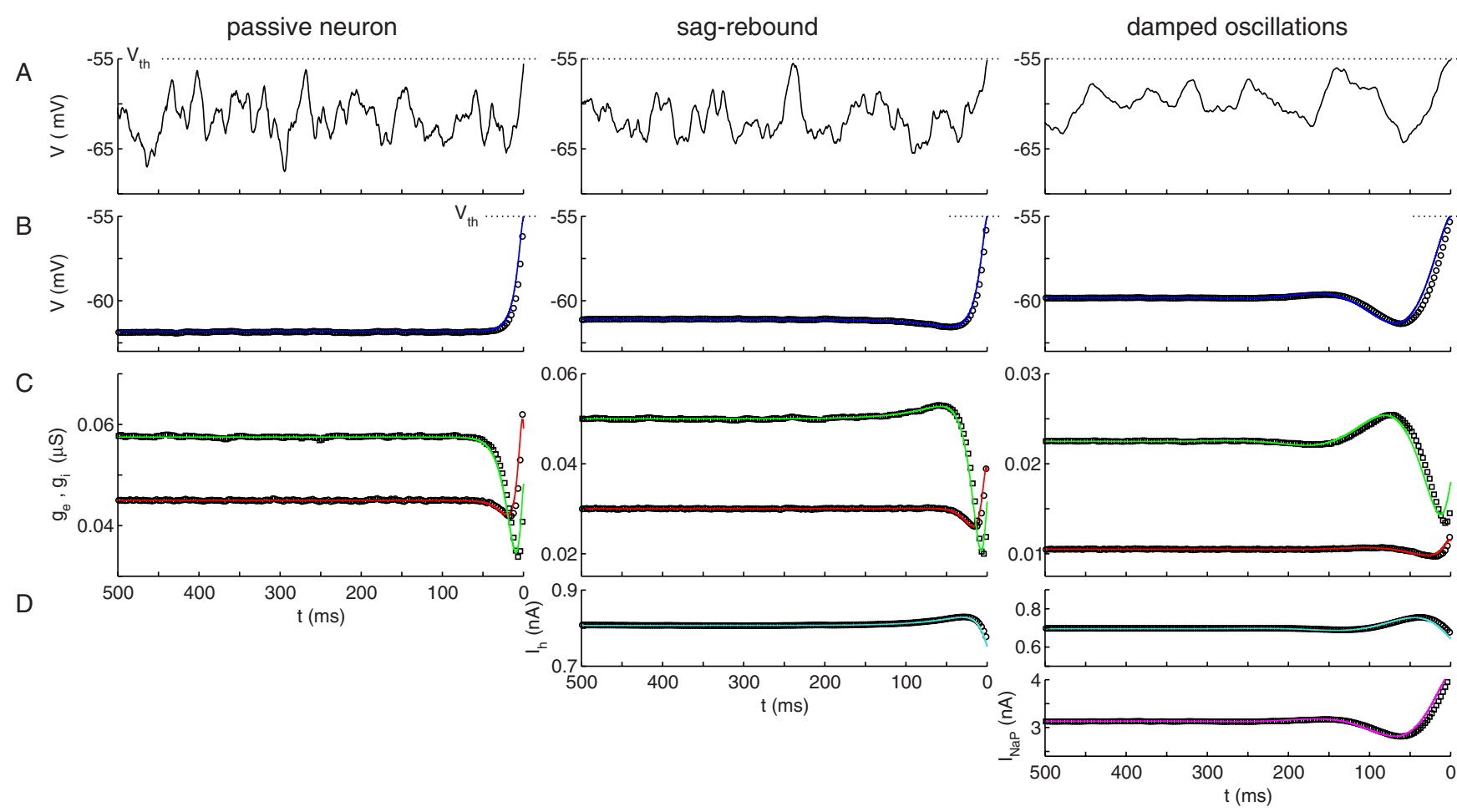

FIG. 4. (Color online) Spike-triggered averages: effects of correlations between excitation and inhibition. The theoretical predictions of Appendix C (solid lines) with numerical simulations of the simplified model (symbols) for a correlation coefficient $\rho=0.4$. (A) Sample voltage trajectories leading to an output spike. (B) Spike-triggered average voltage. (C) Spike-triggered average synaptic conductances (circles: excitatory conductance; squares: inhibitory conductance). (D) Spike-triggered average values of the intrinsic membrane current (circles: $I_{h}, h$-current; squares: $I_{\mathrm{NaP}}$, persistent sodium current). The parameters are the same as in Fig. 2 , except $\sigma_{x}=4.86 \mathrm{mV}, \sigma_{y}$ $=3.33 \mathrm{mV}$, firing rate $0.7 \mathrm{~Hz}$ (decay), $\sigma_{x}=3.27 \mathrm{mV}, \sigma_{y}=2.90 \mathrm{mV}$, firing rate $0.1 \mathrm{~Hz}$ (sag-rebound), and $\sigma_{x}=5.84 \mathrm{mV}, \sigma_{y}=5.89 \mathrm{mV}$, firing rate $0.2 \mathrm{~Hz}$ (damped oscillations).

\section{Including cross correlations between excitation and inhibition}

The theory developed above is readily extended to the case of correlated synaptic drive, in which the excitatory and inhibitory conductances are no longer independent. Details of how this extension can be implemented are given in Appendix C. Figure 4 shows an example of the effects of correlated synaptic fluctuations, for which the correlation coefficient $\rho$ is equal to 0.4 (see Appendix for a definition) where a positive correlation coefficient means that excitatory and inhibitory inputs tend to occur synchronously, thus partially canceling each other. In this case, the theory again predicts spike-triggered average quantities very satisfactorily, with the discrepancy near the threshold comparable to the uncorrelated case.

Although the time course of the membrane voltage is largely unaffected by the correlations, the shape of the synaptic conductances is significantly altered. Technically, this difference is due to the appearance of a mixed mode $\sim e^{t / \tau_{y}}$ in the excitatory conductance, and vice versa for inhibition. Some modes may be suppressed and others enhanced in a way that will be most favorable for the emission of a spike, leading to significant changes in the patterns of the spiketriggered average synaptic inputs. For example, in the case of passive decay, the initial slow decrease in inhibition is accompanied by a simultaneous decrease in excitation as a result of the correlation between the two. The fast increase in excitation that follows is also accompanied by an increase in the inhibitory conductance. Finally, it can be noted that the case of negative correlations does not differ significantly from the uncorrelated case and is thus not examined in detail here.

\section{DISCUSSION}

We have derived analytical expressions for the spiketriggered average of generalized integrate-and-fire neurons with voltage-activated subthreshold currents. Our theory allowed us to construct a direct relation between spiketriggered averages and neuronal response properties, complementing other approaches that focused on the relation to different quantities such as the population activity [11] or the phase response curve [37]. Our results, which are exact in the limit of low-firing rates, are shown to provide a good approximation to the empirical spike-triggered average for firing rates up to a few $\mathrm{Hz}$. The model used in this paper is able to reproduce three important types of subthreshold voltage dynamics seen in biological neurons: passive decay, $h$-current sag, and damped voltage oscillations. The results clearly demonstrate that the form of the spike-triggered average is determined by both the response properties of the neuron and the statistics of the synaptic input, with potential implications for models of spike time-dependent plasticity 
where the spike-triggered average membrane potential plays a role in shaping the distribution of synaptic weights.

Although other parameters, such as conductance and shotnoise effects, the nonlinearity of voltage-gated conductances, or the nature of the spike-generation mechanism, may have an influence on the precise shape of the STV, they do not change the qualitative aspect of our results. Therefore, our analysis also emphasizes the potential of the generalized integrate-and-fire model as an analytical tool: the results presented here are in good agreement with numerical simulations of the full nonlinear model comprising two voltagegated currents and driven by conductance-based synaptic shot noise. Our calculations show that for these type of models, the form of the spike-triggered average depends on the response properties of the neuron in the vicinity of the mean voltage, which could underlie a switching between different modes of information processing depending on the amount of background synaptic input. As an example, stellate cells of the entorhinal cortex display sag-rebound behavior at resting and hyperpolarized membrane potentials, and damped oscillations at more depolarized voltages [22]. This implies that the most likely voltage trajectory has different properties in these voltage ranges, and may support the existence of two distinct operating modes where the neuron fires in response to different types of synaptic inputs.

The path-integral formalism reviewed here has had previous applications for the case of one-variable nonlinear integrate-and-fire neurons [35] and generalized integrateand-fire neurons [14] driven by white noise. Here, we developed an extension of this method to include temporal correlations in the synaptic inputs, which allowed us to separate the roles of excitatory and inhibitory conductances in shaping the membrane potential run-up to the spike. In the regime considered here, i.e., the case of low-frequency, noise-driven spike generation, the average synaptic input consists of an increase in excitation coincident with a withdrawal of inhibition. Since the contribution of each synaptic pathway to the total membrane depolarization scales with the intensity of its fluctuations, the role of inhibition may be as large, or even larger, as that of excitation itself. This shows that inhibitory fluctuations that weaken the background level of inhibition, take an active part in the generation of action potentials- $-\mathrm{a}$ fact that could be particularly important during highconductance, cortical up states.

\section{ACKNOWLEDGMENTS}

This research was supported by a grant from the Swiss National Science Foundation. M.J.E.R. is supported by the Research Councils United Kingdom.

\section{APPENDIX A: REDUCTION OF THE CONDUCTANCE- BASED MODEL}

The reduction strategy is to consider the voltage fluctuations as small deviations away from the resting potential $E_{0}$, which will be determined below. Expansion of the shot-noise synaptic conductances leads to a Gaussian approximation of the fluctuations and expansion voltage-activated currents leads to a linear description of the membrane response.

\section{Gaussian approximation of the synaptic current}

Following [27], the synaptic conductances (using excitation as an example) in Eq. (5) can be separated into tonic $g_{e 0}$ and fluctuating $g_{e F}$ components $g_{e}=g_{e 0}+g_{e F}$, where $g_{e 0}$ $=c_{e} \tau_{x} R_{e}$. In the diffusion approximation the shot-noise conductance fluctuations become an Ornstein-Uhlenbeck process

$$
\tau_{x} \frac{d g_{e F}}{d t}=-g_{e F}+\sigma_{e} \sqrt{2 \tau_{x}} \xi(t),
$$

where the conductance variance is $\sigma_{e}^{2}=c_{e}^{2} \tau_{x} \mathcal{R}_{e} / 2$ and $\xi(t)$ is zero-mean, delta-correlated $\left\langle\xi(t) \xi\left(t^{\prime}\right)\right\rangle=\delta\left(t-t^{\prime}\right)$ Gaussian white noise. The fluctuations $g_{e F}(t)$ and $g_{i F}(t)$ drive the voltage away from its rest $E_{0}$ so the synaptic current [Eq. (5)] may be approximated to first order in $V-E_{0}$ as

$I_{s y n} \simeq g_{e 0}\left(E_{e}-V\right)+g_{i 0}\left(E_{i}-V\right)+g_{e F}\left(E_{e}-E_{0}\right)+g_{i F}\left(E_{i}-E_{0}\right)$.

Terms of the form $g_{e F}\left(E_{0}-V\right)$, and similar for inhibition, have been dropped because they are beyond first order: $V$ $-E_{0}$ grows with $g_{e F}$ and $g_{i F}$.

\section{Linearization of voltage-gated currents}

A convenient method [24,25] is to linearize the voltage around resting potential $E_{0}$, which in this context is the steady-state voltage of the neuron in the absence of synaptic fluctuations (though retaining the tonic conductance of the synaptic drive). The potential $E_{0}$ can be found from the numerical solution of the following equation:

$$
E_{0}=\frac{g_{L} E_{L}+g_{W} W_{0} E_{W}+g_{P} P_{0} E_{P}+g_{e 0} E_{e}+g_{i 0} E_{i}}{g_{L}+g_{W} W_{0}+g_{P} P_{0}+g_{e 0}+g_{i 0}},
$$

where $W_{0}=W_{\infty}\left(E_{0}\right)$ and $P_{0}=P_{\infty}\left(E_{0}\right)$ denotes the activation variables evaluated at $E_{0}$.

It proves convenient to introduce a variable that measures the voltage deviation from rest $v=V-E_{0}$ and similarly a variable $w=\left(W_{0}-W\right) / W_{0}^{\prime}$ that measures the deviation of the $I_{W}$ activation variable from its resting value $W_{0}$, where $W_{0}^{\prime}$ is the voltage derivative of $W_{\infty}$ evaluated at $E_{0}$.

$$
\begin{aligned}
C \frac{d v}{d t}= & -v g+w g_{W} W_{0}^{\prime}\left(E_{W}-E_{0}\right)+g_{e F}\left(E_{e}-E_{0}\right) \\
& +g_{e F}\left(E_{e}-E_{0}\right), \\
\tau_{w} \frac{d w}{d t} & =v-w,
\end{aligned}
$$

where the conductance $g$ is found to be

$$
g=g_{L}+g_{W} W_{0}+g_{P} P_{0}+g_{P} P_{0}^{\prime}\left(E_{P}-E_{0}\right)+g_{e 0}+g_{i 0} .
$$

These equations, together with the equations for the Gaussian conductance fluctuations of the form (A1), represent the 
first-order expansion of the dynamics. The substitutions $x$ $=\left(g_{e F} / g\right)\left(E_{e}-E_{0}\right), \sigma_{x}=\left(\sigma_{e} / g\right)\left(E_{e}-E_{0}\right), \tau_{x}=\tau_{e}$ and likewise for inhibition and the variable $y$, together with the identifications $\tau_{v}=C / g$ and $\gamma=-\left(g_{W} / g\right) W_{0}^{\prime}\left(E_{W}-E_{0}\right)$ yield the reduced set of four equations (7)-(10).

\section{APPENDIX B: MINIMIZATION OF THE ACTION FUNCTIONAL FOR COLORED NOISE}

We consider the $n$-variables generalized integrate-and-fire model defined by

$$
\begin{gathered}
\tau_{v} \frac{d v}{d t}=-v-\sum_{k=1}^{n-1} \gamma_{k} w_{k}+I(t), \\
\tau_{k} \frac{d w_{k}}{d t}=v-w_{k},
\end{gathered}
$$

for $k=1, \ldots, n-1$, where $I(t)=x(t)+y(t)$ is the fluctuating part of the synaptic current. The most probable escape trajectory for this system is obtained by minimizing the action functional

$$
\mathcal{S}(x, y)=\frac{1}{4} \int_{-\infty}^{0}\left\{\frac{1}{\sigma_{x}^{2} \tau_{x}}\left(\mathcal{L}_{x} x\right)^{2}+\frac{1}{\sigma_{y}^{2} \tau_{y}}\left(\mathcal{L}_{y} y\right)^{2}\right\} d t .
$$

In order to perform the minimization, it is necessary to calculate the response of the model to an injected current $I(t)$. We start by rewriting the system in vector notation as

$$
\dot{u}=A u+J(t)
$$

where $u=\left(u_{1}, u_{2}, \ldots, u_{n}\right)$ is a vector containing the voltage variable $\left(u_{1}=v\right)$ and the $n-1$ auxiliary variables $\left(u_{k+1}\right.$ $\left.=w_{k}, k=1, \ldots, n-1\right)$, and $J(t)=\left(I(t) / \tau_{v}, 0,0, \ldots, 0\right)$. The ma$\operatorname{trix} A$ is formed of the coefficients of the linear system. If the time constants $\tau_{k}$ are all different, this matrix admits $n$ distinct eigenvectors $e_{1}, \ldots, e_{n}$ (generally complex valued), associated with the eigenvalues $\lambda_{1}, \ldots, \lambda_{n}$. The change of variables $z=S^{-1} u$, where $S=\left(e_{1}, \ldots, e_{n}\right)$ is the matrix whose columns are formed of the eigenvectors of $A$, results in the diagonalized system

$$
\dot{z}=D z+\widetilde{J}(t),
$$

where $D=\operatorname{diag}\left(\lambda_{1}, \ldots, \lambda_{n}\right)$, and $\widetilde{J}=S^{-1} J$. This system is easily solved and the vector $u$ is obtained with the inverse transformation $u=S z$. For an initial condition of the form $u(0)$ $=\left(u_{1}^{0}, u_{2}^{0}, \ldots, u_{n}^{0}\right)$, this gives

$$
u_{i}(t)=\sum_{k, l} S_{i k} S_{k l}^{-1} u_{l}^{0} e^{\lambda_{k} t}+\int_{0}^{t} \sum_{k} P_{i k} e^{\lambda_{k}(t-s)} \frac{I(s)}{\tau_{v}} d s,
$$

where the coefficients $P_{i k}$ are given by $P_{i k}=S_{i k} S_{k 1}^{-1}$. Thus, for a trajectory starting at the equilibrium point $u^{0}$ $=(0,0, \ldots, 0)$ at time $-\infty$, the threshold condition can be written as

$$
\mathcal{G}(x, y)=\int_{-\infty}^{0} \sum_{k} p_{k} e^{-\lambda_{k} t}[x(t)+y(t)] d t-\tau_{v} v_{\theta}=0,
$$

which is the $n$-dimensional analog of Eq. (33). Note that we have written $p_{k}$ instead of $P_{1 k}$. The first extremality condition (Euler-Lagrange equation) thus reads

$$
\left(1-\tau_{x}^{2} \frac{d^{2}}{d t^{2}}\right) x(t)-\Lambda \sum_{k} p_{k} e^{-\lambda_{k} t}=0 .
$$

This equation is solved by

$$
x(t)=\sum_{k=1}^{n+1} c_{k} e^{-\lambda_{k} t},
$$

where we have defined $\lambda_{n+1}=-1 / \tau_{x}$. Similarly, we obtain for $y(t)$,

$$
y(t)=\sum_{k=1}^{n+1} d_{k} e^{-\nu_{k} t}
$$

where $\vec{\nu}=\left(\lambda_{1}, \ldots, \lambda_{n},-1 / \tau_{y}\right)$.

When these expressions are inserted back into the action functional and threshold condition, we obtain an algebraic minimization problem for the constants $c_{k}$ and $d_{k}$. The solution can be written in matrix form as

$$
\begin{gathered}
\Lambda=\frac{\tau_{v} v_{\theta}}{\sigma_{x}^{2} \tau_{x}\left(\vec{\mu}^{T} X^{-1} \vec{\mu}\right)+\sigma_{y}^{2} \tau_{y}\left(\vec{\eta}^{T} Y^{-1} \vec{\eta}\right)}, \\
\vec{c}=\left(\Lambda \sigma_{x}^{2} \tau_{x}\right) X^{-1} \vec{\mu}, \\
\vec{d}=\left(\Lambda \sigma_{y}^{2} \tau_{y}\right) Y^{-1} \vec{\eta},
\end{gathered}
$$

where for $k, l=1, \ldots, n+1$,

$$
\begin{gathered}
X_{k l}=-\frac{\left(1-\tau_{x} \lambda_{k}\right)\left(1-\tau_{x} \lambda_{l}\right)}{\lambda_{k}+\lambda_{l}}, \\
\mu_{k}=-\sum_{i=1}^{n} \frac{p_{i}}{\lambda_{i}+\lambda_{k}},
\end{gathered}
$$

and similar expressions for $Y$ and $\eta$ are obtained by switching the indices $x$ and $y$ and the eigenvalues $\lambda_{k}$ and $\nu_{k}$. Finally, the amount $\theta_{x}$ of membrane depolarization that is contributed by the excitatory drive can be written as

$$
\theta_{x}=v_{\theta}\left(1+\frac{\sigma_{y}^{2} \tau_{y}\left(\vec{\eta}^{T} Y^{-1} \vec{\eta}\right)}{\sigma_{x}^{2} \tau_{x}\left(\vec{\mu}^{T} X^{-1} \vec{\mu}\right)}\right)^{-1}
$$

with a similar expression for $\theta_{y}$.

Using the notations of Appendix A, the time course of the synaptic conductances $g_{e}(t)=g_{e 0}+x(t) /\left(E_{e}-E_{0}\right)$ and $g_{i}(t)$ $=g_{i 0}+y(t) /\left(E_{i}-E_{0}\right)$ are determined from the constants $c_{k}$ and $d_{k}$ and Eqs. (B9) and (B10). The membrane voltage is then obtained using Eq. (B6). For $n=2$, we obtain Eqs. (34) and (35) for the synaptic conductances, and $v(t)=v_{x}(t)+v_{y}(t)$, where 


$$
\begin{aligned}
v_{x}(t)= & -\left(\frac{p_{1}}{2 \lambda_{1}}+\frac{p_{2}}{\lambda_{1}+\lambda_{2}}\right) \frac{c_{1}}{\tau_{v}} e^{-\lambda_{1} t}-\left(\frac{p_{1}}{\lambda_{1}+\lambda_{2}}+\frac{p_{2}}{2 \lambda_{2}}\right) \frac{c_{2}}{\tau_{v}} e^{-\lambda_{2} t} \\
& +\left(\frac{\tau_{x} p_{1}}{1-\tau_{x} \lambda_{1}}+\frac{\tau_{x} p_{2}}{1-\tau_{x} \lambda_{2}}\right) \frac{c_{3}}{\tau_{v}} e^{t / \tau_{x}},
\end{aligned}
$$

and $v_{y}$ is obtained from $v_{x}$ by switching the indices $x$ and $y$. The coefficients $p_{k}=P_{1 k}$ are those introduced in Eq. (B6) and for the voltage variable we have

$$
p_{1}=\frac{1+\tau_{w} \lambda_{1}}{\tau_{w}\left(\lambda_{1}-\lambda_{2}\right)}, \quad p_{2}=\frac{1+\tau_{w} \lambda_{2}}{\tau_{w}\left(\lambda_{2}-\lambda_{1}\right)},
$$

which gives Eq. (36). The second subthreshold current $w(t)$ is obtained with Eq. (B17) using the coefficients

$$
p_{1}=-p_{2}=\frac{\tau_{v}\left(1+\tau_{w} \lambda_{1}\right)\left(1+\tau_{w} \lambda_{2}\right)}{\gamma \tau_{w}^{2}\left(\lambda_{2}-\lambda_{1}\right)} .
$$

This gives $w(t)=w_{x}(t)+w_{y}(t)$, where

$$
\begin{aligned}
w_{x}(t)= & \theta_{x}\left(\frac{\left(1-\tau_{x} \lambda_{1}\right)\left(1-\tau_{x} \lambda_{2}\right)\left(1+\tau_{w} \lambda_{1}\right)\left(1+\tau_{w} \lambda_{2}\right)}{\gamma \tau_{w}\left[1+\tau_{w}^{2} \lambda_{1} \lambda_{2}-\tau_{x}\left(\lambda_{1}+\lambda_{2}\right)\right]}\right) \\
& \times\left(\frac{\tau_{v} \lambda_{2}\left(1+\tau_{w} \lambda_{1}\right)}{\left(1-\tau_{x}^{2} \lambda_{1}^{2}\right)\left(\lambda_{2}-\lambda_{1}\right)} e^{-\lambda_{1} t}\right. \\
& +\frac{\tau_{v} \lambda_{1}\left(1+\tau_{w} \lambda_{2}\right)}{\left(1-\tau_{x}^{2} \lambda_{2}^{2}\right)\left(\lambda_{1}-\lambda_{2}\right)} e^{-\lambda_{2} t} \\
& \left.+\frac{\left(\tau_{v}+\tau_{w}\right)\left(\tau_{x}-\tau_{w}\right) \tau_{x}^{2} \lambda_{1} \lambda_{2}}{\left(1-\tau_{x}^{2} \lambda_{1}^{2}\right)\left(1-\tau_{x}^{2} \lambda_{2}^{2}\right)} e^{t / \tau_{x}}\right) .
\end{aligned}
$$

Finally, the time course of the intrinsic membrane currents in Fig. 1 can be calculated using the parameters from the linearization of the conductance-based model as

$$
\begin{gathered}
I_{W}(t)=-g_{W}\left(W_{\infty}\left(E_{0}\right)+\left.w(t) \frac{d W_{\infty}}{d v}\right|_{E_{0}}\right)\left(E_{0}-E_{W}\right), \\
I_{P}(t)=-g_{P}\left(P_{\infty}\left(E_{0}\right)+\left.\left(v(t)-E_{0}\right) \frac{d P_{\infty}}{d v}\right|_{E_{0}}\right)\left(E_{0}-E_{P}\right) .
\end{gathered}
$$

\section{APPENDIX C: CROSS CORRELATIONS BETWEEN SYNAPTIC EXCITATION AND INHIBITION}

Correlations between the two synaptic inputs can be modeled by defining the synaptic input as

$$
\begin{gathered}
\tau_{x} \frac{d x}{d t}=-x+\sigma_{x} \sqrt{2 \tau_{x}}\left[\sqrt{1-\rho^{2}} \xi_{x}(t)-\rho \xi_{y}(t)\right], \\
\tau_{y} \frac{d y}{d t}=-y+\sigma_{y} \sqrt{2 \tau_{y}} \xi_{y}(t),
\end{gathered}
$$

where $\rho$ is the cross-correlation coefficient between the excitatory and inhibitory inputs (not the synaptic conductances themselves). In this case, the action functional takes the form

$$
\begin{aligned}
\mathcal{S}(x, y)= & \frac{1}{4(1+\rho)} \int_{-\infty}^{0}\left\{\frac{\left(\mathcal{L}_{x} x\right)^{2}}{\sigma_{x}^{2} \tau_{x}}+\frac{\left(\mathcal{L}_{y} y\right)^{2}}{\sigma_{y}^{2} \tau_{y}}\right. \\
& \left.+2 \rho \frac{\left(\mathcal{L}_{x} x\right)\left(\mathcal{L}_{y} y\right)}{\sqrt{\sigma_{x}^{2} \tau_{x}} \sqrt{\sigma_{y}^{2} \tau_{y}}}\right\} d t .
\end{aligned}
$$

The associated Euler-Lagrange equations read

$$
\begin{aligned}
& \frac{\mathcal{L}_{x}^{\dagger} \mathcal{L}_{x} x}{\sigma_{x}^{2} \tau_{x}}+\rho \frac{\mathcal{L}_{x}^{\dagger} \mathcal{L}_{y} y}{\sqrt{\sigma_{x}^{2} \tau_{x}} \sqrt{\sigma_{y}^{2} \tau_{y}}}=\Lambda \sum_{k} p_{k} e^{-\lambda_{k} t}, \\
& \frac{\mathcal{L}_{y}^{\dagger} \mathcal{L}_{y} y}{\sigma_{y}^{2} \tau_{y}}+\rho \frac{\mathcal{L}_{y}^{\dagger} \mathcal{L}_{x} x}{\sqrt{\sigma_{x}^{2} \tau_{x}} \sqrt{\sigma_{y}^{2} \tau_{y}}}=\Lambda \sum_{k} p_{k} e^{-\lambda_{k} t},
\end{aligned}
$$

and are solved by

$$
\begin{aligned}
& x(t)=\sum_{k=1}^{n+2} c_{k} e^{-\lambda_{k} t}, \\
& y(t)=\sum_{k=1}^{n+2} d_{k} e^{-\lambda_{k} t},
\end{aligned}
$$

with $\lambda_{n+1}=-1 / \tau_{x}$ and $\lambda_{n+2}=-1 / \tau_{y}$. This leads to the coupled system of algebraic equations

$$
\left(\begin{array}{cc}
X & Z \\
Z^{T} & Y
\end{array}\right)\left(\begin{array}{l}
\vec{c} \\
\vec{d}
\end{array}\right)=\Lambda\left(\begin{array}{c}
\vec{\mu} \\
\vec{\eta}
\end{array}\right),
$$

where the matrices $X, Y, Z$ are defined by

$$
\begin{gathered}
X_{k l}=-\frac{\left(1-\tau_{x} \lambda_{k}\right)\left(1-\tau_{x} \lambda_{l}\right)}{\left(\sigma_{x}^{2} \tau_{x}\right)\left(\lambda_{k}+\lambda_{l}\right)}, \\
Y_{k l}=-\frac{\left(1-\tau_{y} \lambda_{k}\right)\left(1-\tau_{y} \lambda_{l}\right)}{\left(\sigma_{y}^{2} \tau_{y}\right)\left(\lambda_{k}+\lambda_{l}\right)}, \\
Z_{k l}=-\rho \frac{\left(1-\tau_{x} \lambda_{k}\right)\left(1-\tau_{y} \lambda_{l}\right)}{\sqrt{\sigma_{x}^{2} \tau_{x}} \sqrt{\sigma_{y}^{2} \tau_{y}}\left(\lambda_{k}+\lambda_{l}\right)}, \\
\mu_{k}=\eta_{k}=-\sum_{i=1}^{n} \frac{p_{i}}{\lambda_{i}+\lambda_{k}} .
\end{gathered}
$$

Using the definition

$$
\left(\begin{array}{c}
\vec{\mu}^{*} \\
\vec{\eta}^{*}
\end{array}\right)=\left(\begin{array}{cc}
X & Z \\
Z^{T} & Y
\end{array}\right)^{-1}\left(\begin{array}{c}
\vec{\mu} \\
\vec{\eta}
\end{array}\right)
$$

we obtain the Lagrange multiplier as

$$
\Lambda=\frac{\tau_{v} v_{\theta}}{\vec{\mu} \vec{\mu}^{*}+\vec{\eta} \vec{\eta}^{*}} .
$$

Finally, the coefficients $c_{k}$ and $d_{k}$ are given by $\vec{c}=\Lambda \vec{\mu}^{*}$ and $\vec{d}=\Lambda \vec{\eta}^{*}$. 
[1] E. de Boer and P. Kuyper, IEEE Trans. Biomed. Eng. BME15, 169 (1968).

[2] J. J. Eggermont, P. I. M. Johannesma, and A. M. H. J. Aersten, Q. Rev. Biophys. 16, 341 (1983).

[3] P. Z. Marmarelis and K. Naka, Science 175, 1276 (1972).

[4] M. Meister, J. Pine, and D. A. Baylor, J. Neurosci. Methods 51, 95 (1994).

[5] R. C. Reid and R. M. Shapley, Nature (London) 356, 716 (1992).

[6] J. P. Jones and L. A. Palmer, J. Neurophysiol. 58, 1187 (1987).

[7] D. Smyth, B. Willmore, G. E. Baker, I. D. Thompson, and D. J. Tolhurst, J. Neurosci. 23, 4746 (2003).

[8] F. E. Theunissen, S. M. N. Woolley, A. Hsu, and T. Frenouw, Ann. N. Y. Acad. Sci. 1016, 187 (2004).

[9] L. Paninski, Network Comput. Neural Syst. 14, 437 (2003).

[10] E. J. Chichilnisky, Network Comput. Neural Syst. 12, 199 (2001).

[11] W. Gerstner, Neural Networks 14, 599 (2001).

[12] B. Agüera y Arcas and A. Fairhall, Neural Comput. 15, 1715 (2003).

[13] J. Kanev, G. Wenning, and K. Obermayer, Neurocomputing 58-60, 47 (2004).

[14] L. Badel, W. Gerstner, and M. J. E. Richardson, Neurocomputing 69, 1062 (2006).

[15] L. Paninski, Neural Comput. 18, 2592 (2006).

[16] H.-C. Pape, Annu. Rev. Physiol. 58, 299 (1996).

[17] B. R. Hutcheon, R. M. Miura, Y. Yarom, and E. Puil, J. Neurophysiol. 71, 583 (1994).

[18] C. T. Dickson, J. Magistretti, M. H. Shalinski, E. Fransén, M. E. Hasselmo, and A. Alonso, J. Neurophysiol. 83, 2562 (2000).

[19] F. G. Pike, R. S. Goddard, J. M. Suckling, P. Ganter, N. Kast- huri, and O. Paulsen, J. Physiol. 529, 205 (2000).

[20] M. J. E. Richardson, N. Brunel, and V. Hakim, J. Neurophysiol. 89, 2538 (2003).

[21] N. Brunel, V. Hakim, and M. J. E. Richardson, Phys. Rev. E 67, 051916 (2003).

[22] E. Fransén, A. A. Alonso, C. T. Dickson, J. Magistretti, and M. E. Hasselmo, Hippocampus 14, 368 (2004).

[23] R. B. Stein, Biophys. J. 7, 37 (1967).

[24] A. L. Hodgkin and A. F. Huxley, J. Physiol. 117, 500 (1952).

[25] C. Koch, Biol. Cybern. 50, 15 (1984).

[26] B. Hutcheon, R. M. Miura, and E. Puil, J. Neurophysiol. 76, 683 (1996).

[27] M. J. E. Richardson and W. Gerstner, Neural Comput. 17, 923 (2005).

[28] M. J. E. Richardson and W. Gerstner, Chaos 16, 026106 (2006).

[29] P. Lansky and V. Lanska, Biol. Cybern. 56, 19 (1987).

[30] A. N. Burkitt and G. M. Clark, Neurocomputing 26-27, 93 (1999).

[31] A. N. Burkitt, Biol. Cybern. 85, 247 (2001).

[32] M. J. E. Richardson, Phys. Rev. E 69, 051918 (2004).

[33] G. La Camera, W. Senn, and S. Fusi, Neurocomputing 58-60, 253 (2004).

[34] M. I. Freidlin and A. D. Wentzell, Random Perturbations of Dynamical Systems (Springer-Verlag, New York, Heidelberg, Berlin, 1984).

[35] L. Paninski, J. Comput. Neurosci. 21, 71 (2006).

[36] A. Destexhe, M. Rudolph, and D. Paré, Nat. Rev. Neurosci. 4, 739 (2003).

[37] G. B. Ermentrout, R. F. Galán, and N. N. Urban, Phys. Rev. Lett. 99, 248103 (2007). 